\title{
Nonlinear self-interference cancellation in MIMO full-duplex transceivers under crosstalk
}

\author{
Dani Korpi* (D, Lauri Anttila and Mikko Valkama
}

\begin{abstract}
This paper presents a novel digital self-interference canceller for an inband multiple-input-multiple-output (MIMO) full-duplex radio. The signal model utilized by the canceller is capable of modeling the in-phase quadrature (IQ) imbalance, the nonlinearity of the transmitter power amplifier, and the crosstalk between the transmitters, thereby being the most comprehensive signal model presented thus far within the full-duplex literature. Furthermore, it is also shown to be valid for various different radio frequency (RF) cancellation solutions. In addition to this, a novel complexity reduction scheme for the digital canceller is also presented. It is based on the widely known principal component analysis, which is used to generate a transformation matrix for controlling the number of parameters in the canceller. Extensive waveform simulations are then carried out, and the obtained results confirm the high performance of the proposed digital canceller under various circuit imperfections. The complexity reduction scheme is also shown to be capable of removing up to $65 \%$ of the parameters in the digital canceller, thereby significantly reducing its computational requirements.
\end{abstract}

Keywords: Full-duplex, MIMO, Self-interference, RF impairments, Crosstalk

\section{Introduction}

Inband full-duplex communications is a promising candidate technology for further improving the spectral efficiency of the next generation wireless systems, such as the $5 \mathrm{G}$ networks [1-11]. The basic idea behind it is to transmit and receive at the same time at the same center-frequency, thereby in principle doubling the spectral efficiency. The drawback of such inband full-duplex operation is the own transmit signal, which is coupling to the receiver and becomes an extremely powerful source of self-interference (SI). The most significant challenge in implementing inband full-duplex radios in practice is thereby the development of SI cancellation solutions, which are capable of removing the SI in the receiver. There are already reports of various demonstrator implementations, which achieve relatively high SI cancellation performance, thereby allowing for true inband full-duplex operation $[1-3,6,7,11-14]$.

Moreover, in order to meet the high throughput requirements of the future wireless networks, it is inevitable that

\footnotetext{
*Correspondence: dani.korpi@tut.fi

Department of Electronics and Communications Engineering, Tampere University of Technology, Tampere, Finland
}

the inband full-duplex concept must be combined with MIMO capabilities in the transceivers [7, 12-19]. This obviously results in a higher physical layer capacity, but it also requires more elaborate SI cancellation solutions. In particular, in a MIMO transceiver, the observed SI signal in each receiver consists of a combination of all the transmit signals, which means that also the SI cancellers must have all of the transmit signals available. Furthermore, in order to perform SI cancellation, the coupling channels between all the transmitters and receivers must be estimated, which results in a somewhat more demanding SI cancellation procedure. Nevertheless, this increased complexity is justified by the higher physical layer throughputs.

Especially the complexity of the RF canceller is heavily affected by the number of transmitters and receivers $[7,15]$. For an $N_{T} \times N_{R}$ MIMO transceiver, the RF canceller requires at least $N_{T} N_{R}$ cancellation paths, or even more if using a multi-tap solution $[7,20]$. This number can be somewhat decreased by using auxiliary transmitters to upconvert digitally generated cancellation signals, since then only $N_{R}$ cancellation paths are required. However, the drawback of this solution is obviously the need for additional RF transmitters, as well as the fact that the 
digitally generated cancellation signals do not include any of the transmitter-induced impairments, which thereby remain unaffected by this type of an RF cancellation solution [1]. Another possible solution for decreasing the complexity of RF cancellation in the context of very large transmit antenna arrays is to use beamforming to form nulls in the receive antennas $[4,21]$, which might even allow for completely omitting RF cancellation. In typical MIMO devices, however, the increase in the RF cancellation complexity is more or less inevitable.

Also, the complexity of digital SI cancellation is somewhat increased under MIMO operation, but it is obviously more straight-forward to process several SI signals in the digital domain. In particular, more computational resources are needed to estimate all the channel responses between the several transmitters and receivers, but no additional RF hardware is required. However, having several transmit chains on a single chip introduces another issue from the perspective of the digital canceller: the crosstalk between the transmitters, which occurs both before and after the power amplifiers (PAs) [22-28]. This phenomenon is illustrated in Fig. 1 for an example case of three transmitters. What makes this an especially cumbersome issue is the fact that typically the PAs introduce significant nonlinear distortion [3, 29]. This, on the other hand, means that nonlinear modeling of the SI is required in the digital canceller, which is very challenging if the PA input is in fact a linear combination of all the original transmit signals, as is the case under crosstalk [26]. Nevertheless, it is still necessary to model the crosstalk, since otherwise the accuracy of the regenerated SI signal is not sufficiently high. This is especially crucial for the emerging massive MIMO transceivers, where the large amount of transmit chains calls for a

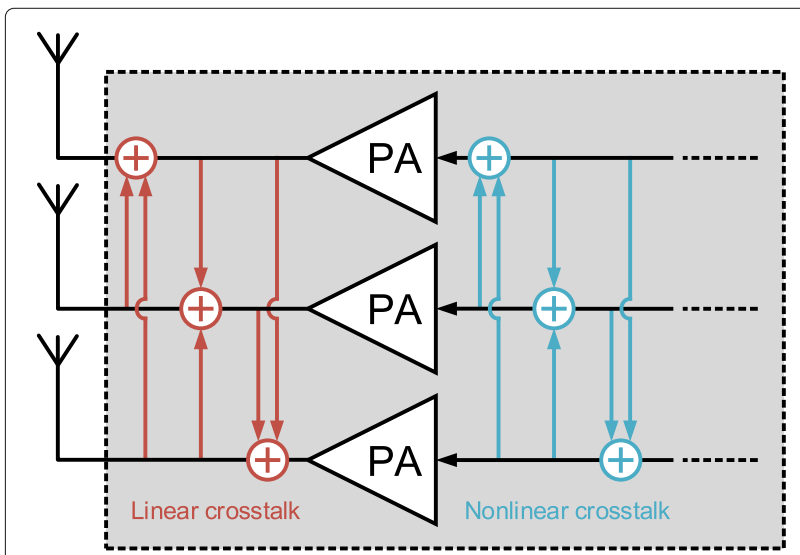

Fig. 1 An illustration of the crosstalk phenomenon in a three-antenna MIMO transmitter, where crosstalk occurs both before and after the PAs. The former is typically referred to as nonlinear crosstalk, while the latter is called linear crosstalk high level of integration, which results in more leakage between the transmission paths [28]. Hence, the increase in computational complexity caused by the crosstalk modeling must be tolerated in order to obtain sufficient levels of SI cancellation also under MIMO operation.

In this article, we present a general signal model for the observed SI in the digital domain under a scenario where there is crosstalk between the transmit chains before and after nonlinear PAs. Moreover, it is shown that the signal model can be applied to various different RF cancellation solutions. The presented comprehensive signal model, which shows the effect of the crosstalk in terms of the original transmit signals, is then used as a basis for a high-performance digital SI canceller. The IQ imbalance occurring both in the transmitters and in the receivers is also included in the signal model, since it is typically one of the dominant sources of distortion in a practical transceiver, alongside with the PA-induced nonlinearities [30].

Furthermore, to address the increase in the computational complexity due to the MIMO operation and crosstalk modeling, a novel principal component analysis (PCA)-based solution is proposed, which can be used to control the complexity of the signal model. In particular, PCA processing is used to identify the insignificant terms in the observed SI signal, which are then omitted in the further cancellation processing. This results in a significant reduction of the unknown parameters that must be estimated, which obviously decreases the computational requirements of the digital SI canceller. Moreover, since the most dominant SI terms are retained by such processing, there is no essential degradation in the cancellation performance. To the best of our knowledge, such complexity reduction schemes have not been previously proposed in the context of SI cancellation solutions.

The detailed list of novel contributions in this paper is as follows:

- We derive the most comprehensive MIMO signal model for the observed SI presented so far in the literature. It covers various RF cancellation scenarios, while also modeling the crosstalk between the transmitters under low-cost nonlinear PAs and IQ imbalance.

- We propose a novel nonlinear digital SI canceller, which utilizes the aforementioned advanced signal model.

- We propose a novel complexity reduction scheme based on PCA, which can be used to control the computational complexity of the digital canceller, while minimizing the decrease in the cancellation performance. 
- We present numerical results, which illustrate various aspects of the proposed digital SI cancellation solution with realistic waveform simulations.

The rest of this article is organized as follows. In Section 2, the MIMO signal model is derived. Then, in Section 3, the actual nonlinear digital SI canceller is presented, alongside with the parameter estimation procedure and the PCA-based complexity reduction scheme. After this, in Section 4, the proposed digital SI cancellation solution is evaluated with realistic waveform simulations. Finally, the conclusions are drawn in Section 5.

\section{Baseband equivalent signal modeling}

In this section, we build a complete SI channel model for a MIMO full-duplex device, including the effects of transmitter impairments (PA nonlinearity, IQ imbalance, and transmitter crosstalk), the linear MIMO SI channel, and RF cancellation. In the forthcoming analysis, the nonlinearities produced by the digital-to-analog and analogto-digital converters (DACs and ADCs) [31], alongside with phase noise, are omitted from the signal model for simplicity, although phase noise is still included in the reported simulation results.

An illustration of the considered full-duplex MIMO transceiver is given in Fig. 2, with two alternative RF cancellation solutions. In particular, the RF cancellation can be done either by utilizing the PA output signals, or by generating the cancellation signals in the digital domain and upconverting them with the help of auxiliary transmitters. In the forthcoming analysis, both of these options are considered. Furthermore, in Fig. 2, the transceiver is shown to have separate transmit and receive antennas only for illustrative purposes, since the same signal model can also be applied to a case where each antenna is shared between a transmitter and a receiver [32]. Hence, the forthcoming analysis is directly applicable also to a shared-antenna architecture. Note that, for notational simplicity, the actual received signals of interest and additive noise are not included in the following presentation.

\subsection{Power amplifier and IQ modulator models with crosstalk}

Let us denote the baseband signal of transmitter $j(j=$ $\left.1,2, \ldots, N_{T}\right)$ by $x_{j}(n)$. The output signal of a frequencyindependent IQ modulator model is [33]

$$
x_{j}^{I Q M}(n)=K_{1, j} x_{j}(n)+K_{2, j} x_{j}^{*}(n)
$$

with $K_{1, j}=1 / 2\left(1+g_{j} \exp \left(j \varphi_{j}\right)\right), K_{2, j}=1 / 2\left(1-g_{j} \exp \left(j \varphi_{j}\right)\right)$, where $g_{j}, \varphi_{j}$ are the gain and phase imbalance parameters of transmitter $j$. Notice that under typical circumstances $\left|K_{1, j}\right| \gg\left|K_{2, j}\right|$. The magnitude of the IQ image component, represented by the conjugated signal term in (1), can be characterized with the image rejection ratio (IRR) as $10 \log _{10}\left(\left|K_{1, j}\right|^{2} /\left|K_{2, j}\right|^{2}\right)$.

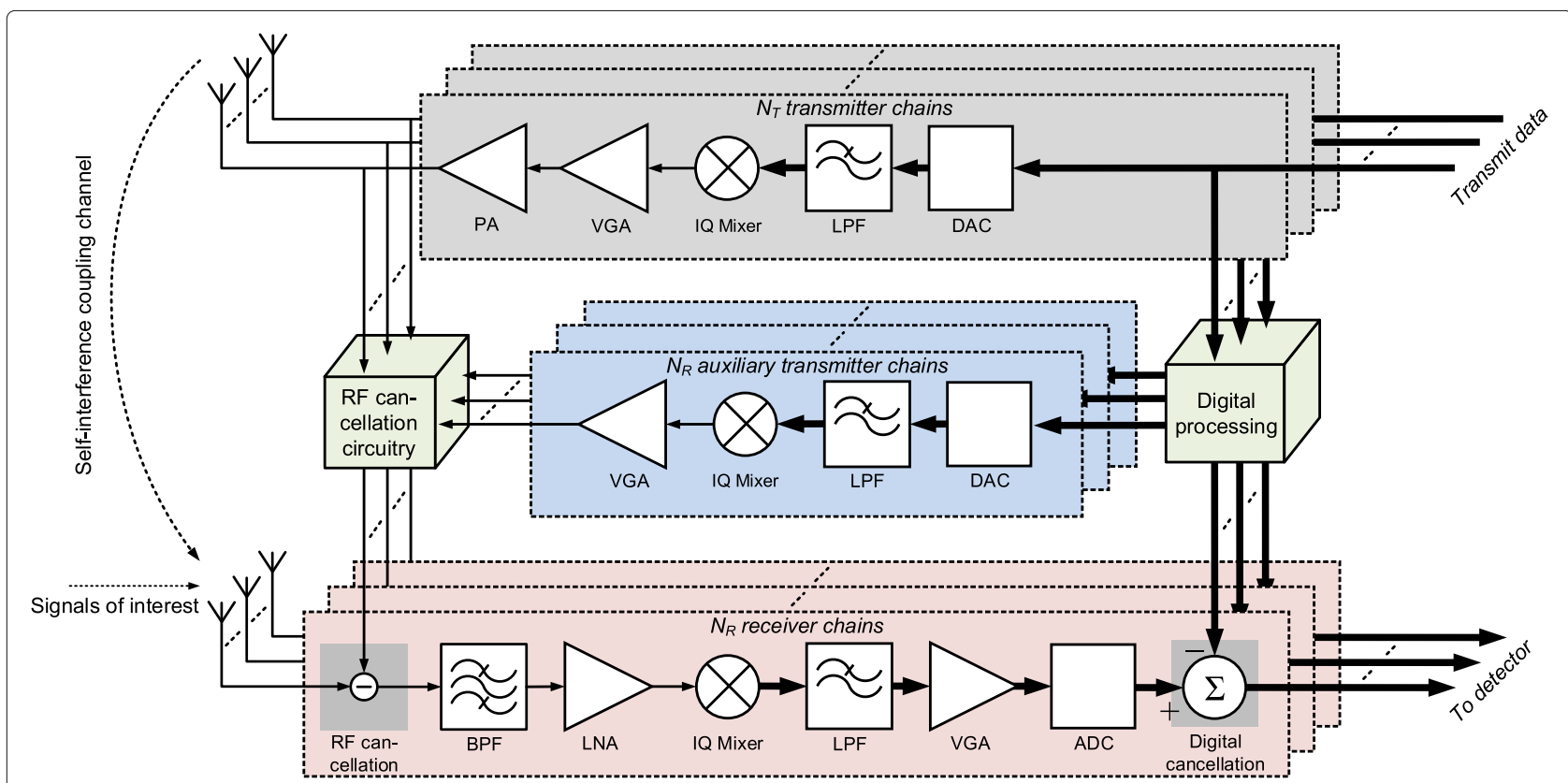

Fig. 2 Block diagram of the considered MIMO full-duplex transceiver with $N_{T}$ transmitters and $N_{R}$ receivers. Two alternative RF cancellation schemes are illustrated in the diagram: one utilizing directly the transmitter output signals and one utilizing auxiliary transmitters with digitally generated RF cancellation signals 
The response of the PA is approximated using the widely known parallel Hammerstein $(\mathrm{PH})$ model, given for transmitter $j$ as [34]

$$
x_{j}^{P A}(n)=\sum_{\substack{p=1 \\ p \text { odd }}}^{P} \sum_{m=0}^{M} h_{p, j}(m) \psi_{p}\left(x_{j, \text { in }}(n-m)\right),
$$

where $x_{j, i n}(n)$ is the PA input signal, the basis functions are defined as

$$
\psi_{p}(x(n))=|x(n)|^{p-1} x(n)=x(n)^{\frac{p+1}{2}} x^{*}(n)^{\frac{p-1}{2}}
$$

and $h_{p, j}(n)$ denote the impulse responses of the $\mathrm{PH}$ branches for transmitter $j$, while $M$ and $P$ denote the memory depth and nonlinearity order of the PH model, respectively [34-36]. The $\mathrm{PH}$ nonlinearity is a widely used nonlinear model for direct as well as inverse modeling of PAs [34-37].

Due to the crosstalk occurring before each PA, referred to as nonlinear crosstalk, the input signal $x_{j, \text { in }}(n)$ can be written as

$$
x_{j, \text { in }}(n)=\sum_{i=1}^{N_{T}} \alpha_{i j} x_{i}^{I Q M}(n)
$$

where $\alpha_{i j}$ is the crosstalk coefficient between the $i$ th and $j$ th transmitter chains, and $\alpha_{j j}=1 \forall j$. In other words, as a result of the crosstalk occurring before the PAs, each PA input signal is in fact a linear combination of all the different transmit signals. The crosstalk phenomenon is illustrated for an example case of three transmitters in Fig. 1, where both the nonlinear and linear crosstalk are shown. Inserting now (1) into (4), we can rewrite the PA input signal as

$$
\begin{aligned}
x_{j, \text { in }}(n) & =\sum_{i=1}^{N_{T}} \alpha_{i j}\left(K_{1, i} x_{i}(n)+K_{2, i} x_{i}^{*}(n)\right) \\
& =\sum_{i=1}^{N_{T}} \alpha_{1, i j} x_{i}(n)+\sum_{i=1}^{N_{T}} \alpha_{2, i j} x_{i}^{*}(n),
\end{aligned}
$$

where $\alpha_{1, i j}=\alpha_{i j} K_{1, i}$ and $\alpha_{2, i j}=\alpha_{i j} K_{2, i}$.
Using (5), the signal at the PA output can be written as follows:

$$
\begin{aligned}
x_{j}^{P A}(n)= & \sum_{\substack{p=1 \\
p \text { odd }}}^{P} \sum_{m=0}^{M} h_{p, j}(m) x_{j, i n}(n-m)^{\frac{p+1}{2}} x_{j, i n}^{*}(n-m)^{\frac{p-1}{2}} \\
= & \sum_{\substack{p=1 \\
p \text { odd }}}^{P} \sum_{m=0}^{M} h_{p, j}(m) \sum_{k_{0}=0}^{\frac{p+1}{2}}\left(\begin{array}{c}
\frac{p+1}{2} \\
k_{0}
\end{array}\right) \\
& \times\left(\sum_{i=1}^{N_{T}} \alpha_{1, i j} x_{i}(n-m)\right)^{\frac{p+1}{2}-k_{0}}\left(\sum_{i=1}^{N_{T}} \alpha_{2, i j} x_{i}^{*}(n-m)\right)^{k_{0}} \\
& \times \sum_{l_{0}=0}^{\frac{p-1}{2}}\left(\begin{array}{c}
\frac{p-1}{2} \\
l_{0}
\end{array}\right)\left(\sum_{i=1}^{\frac{p-1}{2}-l_{0}} \alpha_{2, i j}^{*} x_{i}(n-m)\right)^{N_{T}} \\
& \times\left(\sum_{i=1}^{N_{T}} \alpha_{1, i j}^{*} x_{i}^{*}(n-m)\right)^{l_{0}}
\end{aligned}
$$

It can be further modified by expanding all the integer powers of the sum signals as shown in the Appendix, which gives

$$
\begin{aligned}
x_{j}^{P A}(n)= & \sum_{\substack{p=1 \\
p \text { odd } \\
q_{0}-r_{1}-\cdots-r_{N_{T}-2}}}^{P} \sum_{q_{1}=0}^{p-q_{0}} \cdots \sum_{q_{N_{T}-1}=0}^{p-q_{0}-\cdots-q_{N_{T}-2}} \sum_{r_{1}=0}^{q_{0}} \sum_{r_{2}=0}^{q_{0}-r_{1}} \cdots \\
& \sum_{r_{N_{T}-1}=0}^{p} \sum_{m=0}^{p, j, q_{0}, \ldots, r_{N_{T}-1}}(m) \\
& \times x_{1}(n-m)^{q_{1}} x_{2}(n-m)^{q_{2}} \ldots \\
& x_{N_{T}}(n-m)^{p-\sum_{i=0}^{N_{T}-1} q_{i}} \\
& \times x_{1}^{*}(n-m)^{r_{1}} x_{2}^{*}(n-m)^{r_{2}} \ldots \\
& x_{N_{T}}^{*}(n-m)^{q_{0}-\sum_{i=1}^{N_{T}-1} r_{i}}
\end{aligned}
$$

where $h_{p, j, q_{0}, \ldots, r_{N_{T}-1}}(m)$ are the coefficients for the basis function of the form $\prod_{i=1}^{N_{T}} x_{i}(n)^{a_{i}} x_{i}^{*}(n)^{b_{i}}$ such that $\sum_{i=1}^{N_{T}}\left(a_{i}+b_{i}\right)=p$. This signal model is of similar form as the one presented in [26], with the exception that the model in (7) also incorporates the effect of IQ imbalance and is thus more complete.

In order to simplify (7), it can be noted that, for the $j$ th transmit signal and the $p$ th nonlinearity order, the signal model contains in fact all the different combinations of the exponents $q_{m}$ and $r_{n}$, under the constraint that their sum is equal to $p$. This means that we can rewrite (7) as 


$$
\begin{aligned}
x_{j}^{P A}(n)= & \sum_{\substack{p=1 \\
p \text { odd }}}^{P} \sum_{\substack{k \\
\mathbf{s}^{k} \|_{1}=p}} \sum_{m=0}^{M} h_{j, p, \mathbf{s}^{k}}(m) \\
& \times \prod_{q=1}^{N_{T}} x_{q}(n-m)^{s_{q}^{k}} x_{q}^{*}(n-m)^{s_{q+N_{T}}^{k}},
\end{aligned}
$$

where $\mathbf{s}^{k}$ is the $k$ th combination of the $2 N_{t} \times 1$ exponent vector $\mathbf{s}, h_{j, p, \mathbf{s}^{k}}(m)$ contains the corresponding coefficients, and $\|\cdot\|_{1}$ denotes the $L^{1}$-norm. Note that all the elements of $\mathbf{s}$ are non-negative integers, as per the signal model. To illustrate its structure, all the variations of $\mathbf{s}$ for $N_{T}=1$ and $P=3$ are written below:

$$
\begin{aligned}
& \mathbf{s}^{1}=\left[\begin{array}{ll}
1 & 0
\end{array}\right]^{T}, \mathbf{s}^{2}=\left[\begin{array}{ll}
0 & 1
\end{array}\right]^{T}, \mathbf{s}^{3}=\left[\begin{array}{ll}
1 & 2
\end{array}\right]^{T} \\
& \mathbf{s}^{4}=\left[\begin{array}{ll}
2 & 1
\end{array}\right]^{T}, \mathbf{s}^{5}=\left[\begin{array}{ll}
3 & 0
\end{array}\right]^{T}, \mathbf{s}^{6}=\left[\begin{array}{ll}
0 & 3
\end{array}\right]^{T}
\end{aligned}
$$

After the PAs, there is typically also some additional crosstalk between the transmitters, referred to as linear crosstalk. Taking also this phenomenon into account, the final output signal for the $j$ th transmitter can be written as

$$
\begin{aligned}
x_{j}^{T X}(n)= & \sum_{l=1}^{N_{T}} \beta_{l j} x_{l}^{P A}(n) \\
= & \sum_{\substack{p=1 \\
p \text { odd }\left\|_{\mathbf{s}^{k}}\right\|_{1}=p}} \sum_{m=0}^{M} \sum_{l=1}^{N_{T}} \beta_{l j} h_{l, p, \mathbf{s}^{k}}(m) \\
& \times \prod_{q=1}^{N_{T}} x_{q}(n-m)^{s_{q}^{k}} x_{q}^{*}(n-m)^{s_{q+N_{T}}^{k}},
\end{aligned}
$$

where $\beta_{l j}$ is the crosstalk coefficient between the $l$ th and $j$ th transmitters. It can be observed that the essential signal model remains the same as in (8), but with modified coefficients written as

$$
\dot{h}_{j, p, \mathbf{s}^{k}}(m)=\sum_{l=1}^{N_{T}} \beta_{l j} h_{l, p, \mathbf{s}^{k}}(m) .
$$

Denoting the MIMO propagation channel impulse response from TX antenna $j$ to $\mathrm{RX}$ antenna $i$ by $c_{i j}(l), l=$ $0,1, \ldots, L$, the received SI signal at RX antenna $i(i=$ $\left.1,2, \ldots, N_{R}\right)$ can now be written as

$$
\begin{aligned}
z_{i}(n)= & \sum_{j=1}^{N_{T}} \sum_{l=0}^{L} c_{i j}(l) x_{j}^{T X}(n-l) \\
= & \sum_{\substack{p=1 \\
p \text { odd }\left\|\mathbf{s}^{k}\right\|_{1}=p}} \sum_{j=1}^{N_{T}} \sum_{l=0}^{L} \sum_{m=0}^{M} c_{i j}(l) \dot{h}_{j, p, \mathbf{s}^{k}}(m) \\
& \times \prod_{q=1}^{N_{T}} x_{q}(n-m-l)^{s_{q}^{k}} x_{q}^{*}(n-m-l)^{s_{q+N_{T}}^{k}} \\
= & \sum_{\substack{p=1 \\
p \text { odd }\left\|_{\mathbf{s}^{k}}\right\|_{1}=p\\
}} \sum_{m=0}^{M+L} \widetilde{h}_{i, p, \mathbf{s}^{k}}^{N_{T}} x_{q}(n-m)^{s_{q}^{k}} x_{q}^{*}(n-m)^{s_{q+N_{T}}^{k}} .
\end{aligned}
$$

Again, the signal model still remains the same as in (8), but with slightly modified coefficients, which are obtained from

$$
\tilde{h}_{i, p, \mathbf{s}^{k}}(m)=\sum_{j=1}^{N_{T}} \sum_{l=0}^{m} c_{i j}(l) \dot{h}_{j, p, \mathbf{s}^{k}}(m-l) .
$$

The new memory length of the received signal model is also increased from $M$ to $M+L$. The input signal of the $i$ th receiver $\left(z_{i}(n)\right)$ is then further processed by the RF canceller and the actual receiver chain. Note that the above signal model in (11) also applies to circulator and electrical balance duplexer-based implementations, where each transmitter and receiver pair share the same antenna [32], and hence it is generic in that respect.

\subsection{RF cancellation}

To ensure an extensive analysis and derivation for the proposed digital cancellation algorithm, we consider three different RF cancellation solutions. The first technique is similar to what has been used, e.g, in [5, 6], and it involves directly tapping the transmitter outputs to obtain the reference signals for RF cancellation. This method is based on purely analog processing, as the whole cancellation procedure is performed in the RF domain. The two other considered methods are based on auxiliary TX chains, which are used to produce the RF cancellation signal from digital baseband samples [1, 38, 39]. We call this latter approach hybrid RF cancellation to distinguish it from purely analog cancellation. Furthermore, we consider both linear and nonlinear preprocessing to be used with this auxiliary transmitter based RF cancellation.

\subsubsection{RF cancellation with transmitter output signals}

In this RF cancellation method, the output of each TX chain is tapped, and subtracted from each of the received 
signals after suitable gain, phase and delay adjustments. These RF cancellers can be either single-tap or multi-tap $[9,40]$, for which reason we denote them with impulse responses $h_{i j}^{R F}(l)$, operating on the TX output signals $x_{j}^{T X}(n)$. The coefficients are obviously chosen such that they model the MIMO coupling channel coefficients in $c_{i j}(n)$ as accurately as possible. The RF cancellation signal for the $i$ th receiver can thus be written as

$$
z_{i}^{c}(n)=\sum_{j=1}^{N_{T}} \sum_{l=0}^{L^{\prime}} h_{i j}^{R F}(l) x_{j}^{T X}(n-l),
$$

where $L^{\prime}$ is the number of taps in the RF canceller. It can be easily shown that the cancellation signal is of similar form as the actual received signal in (11), with coefficients of the form

$$
\check{h}_{i, p, \mathbf{s}^{k}}^{R F}(m)=\sum_{j=1}^{N_{T}} \sum_{l=0}^{m} h_{i j}^{R F}(l) \dot{h}_{j, p, \mathbf{s}^{k}}(m-l)
$$

and a memory length of $M+L^{\prime}$. Thus, the received SI signal of receiver $i$, after this type of analog RF cancellation, becomes

$$
\begin{aligned}
r_{i}(n)= & z_{i}(n)-z_{i}^{c}(n) \\
= & \sum_{\substack{p=1 \\
p \text { odd }\left\|\mathbf{s}^{k}\right\|_{1}^{k}}} \sum_{m=p}^{M+\max \left(L, L^{\prime}\right)}\left(\widetilde{h}_{i, p, \mathbf{s}^{k}}(m)\right. \\
& \left.-\check{h}_{i, p, \mathbf{s}^{k}}^{R F}(m)\right) \prod_{q=1}^{N_{T}} x_{q}(n-m)^{s_{q}^{k}} x_{q}^{*}(n-m)^{s_{q+N_{T}}^{k},}
\end{aligned}
$$

Hence, the structure of the RF canceller output signal model is still of the same form as in (11), but with modified coefficients expressed as $\breve{h}_{i, p, \mathbf{s}^{k}}(m)=\widetilde{h}_{i, p, \mathbf{s}^{k}}(m)-$ $\check{h}_{i, p, \mathbf{s}^{k}}^{R F}(m)$.

This type of purely analog RF cancellation calls for $N_{T} \times$ $N_{R}$ canceller circuits to be implemented in the device, one canceller from each transmitter to each receiver. The complexity may become prohibitive when the number of antennas is significantly increased and, thereby, when implementing a high order full-duplex MIMO device, alternative methods for RF cancellation might have to be considered.

\subsubsection{Hybrid RF cancellation using auxiliary transmitters with linear preprocessing}

One such alternative RF canceller structure is the hybrid method, which utilizes extra transmitter chains, one for each receiver, to upconvert and subtract estimated replicas of the SI signals from the received signals at RF $[1,38,39]$. In this case, linear MIMO filtering is already done at digital baseband on the transmit signals $x_{j}(n)$ with some estimated MIMO channel responses $h_{i j}^{R F}(l)$. Since the transmit signals from the different antennas can now be combined already in the digital domain, the analog hardware complexity of this type of an RF cancellation scheme scales with $N_{R}$ instead of $N_{T} N_{R}$, and may prove to be more attractive with a high number of antennas. Note that in this subsection, we consider only linear processing for the hybrid RF canceller, and thereby IQ modulator imbalance or PA nonlinearity are not explicitly dealt with at this stage. The RF cancellation signal can in this case be written as

$$
z_{i}^{c}(n)=\sum_{j=1}^{N_{T}} \sum_{l=0}^{L^{\prime}} h_{i j}^{R F}(l) x_{j}(n-l),
$$

which is a special case of the signal model in (11) with $P=1$ and coefficients $\breve{h}_{i, 1, \mathbf{s}^{k}}^{R F}(m)$ consisting of $h_{i j}^{R F}(l)$ with proper $\mathbf{s}^{k}$. The signal after RF cancellation is again obtained as shown in (13), and with the final coefficients as

$$
\begin{aligned}
& \breve{h}_{i, p, \mathbf{s}^{k}}(m)=\widetilde{h}_{i, p, \mathbf{s}^{k}}(m)-\breve{h}_{i, p, \mathbf{s}^{k}}^{R F}(m), p=1 \\
& \breve{h}_{i, p, \mathbf{s}^{k}}(m)=\widetilde{h}_{i, p, \mathbf{s}^{k}}(m), p \geq 3
\end{aligned}
$$

Also this model is essentially of the same form as (11), with the coefficients of the linear SI terms being affected by the hybrid RF cancellation procedure, while the other terms remain unchanged. This means that the observed SI signal in the receiver digital domain can still be modeled with the same signal model as in the case of pure analog RF cancellation (or no RF cancellation at all). Thus, from the perspective of the digital cancellation algorithm, it makes no difference whether RF cancellation is performed by tapping the transmitter output or by using auxiliary TX chains with linear preprocessing, although the RF cancellation performance itself might obviously be different for the considered methods.

\subsubsection{Hybrid RF cancellation using auxiliary transmitters with nonlinear preprocessing}

Yet another alternative RF cancellation technique utilizes auxiliary transmitters, but with nonlinear preprocessing, instead of purely linear processing. The estimated MIMO channel responses of the different nonlinear SI terms are now denoted by $h_{i j, p}^{R F}(l)$. In the forthcoming analysis, it is assumed that the auxiliary TX chains are linear. This is a relatively feasible assumption, since no PA is required due to the lower output power requirements. Now, the 
cancellation signal obtained with this RF cancellation procedure can be expressed as

$$
\begin{aligned}
z_{i}^{c}(n)= & \sum_{j=1}^{N_{T}} \sum_{\substack{p=1 \\
p \text { odd }}}^{P^{\prime}} \sum_{l=0}^{L^{\prime}} h_{i j, p}^{R F}(l) x_{j}(n-l)^{\frac{p+1}{2}} \\
& \times x_{j}^{*}(n-l)^{\frac{p-1}{2}},
\end{aligned}
$$

where $P^{\prime}$ is the nonlinearity order of the RF cancellation signals. Note that this signal model neglects IQ imbalance and crosstalk, since the RF canceller must only attenuate the SI such that the receiver is not saturated. Also this RF cancellation signal can be easily represented with a signal model of the same form as in (11). The coefficients $\breve{h}_{i, p, \mathbf{s}^{k}}^{R F}(m)$ of the signal model now consist of $h_{i j, p}^{R F}(l)$ with the parameters $p$ and $\mathbf{s}^{k}$ that correspond to the basis functions $x_{j}(n-l)^{\frac{p+1}{2}} x_{j}^{*}(n-l)^{\frac{p-1}{2}}$, and other coefficients are set to zero. Similar to the other RF cancellation schemes, after subtracting the cancellation signal from the received signal, as in (13), the signal model remains the same and its coefficients are $\breve{h}_{i, p, \mathbf{s}^{k}}(m)=\widetilde{h}_{i, p, \mathbf{s}^{k}}(m)-\breve{h}_{i, p, \mathbf{s}^{k}}^{R F}(m)$. Now, also some of the nonlinear SI terms are attenuated by RF cancellation, as they are modeled in the preprocessing stage.

Overall, it can be concluded that the essential structure of the observed SI signal in the digital domain is independent of the chosen method for RF cancellation. This means that, in the forthcoming analysis, the same digital cancellation algorithm can be applied in all the situations since the only difference between the three alternative RF cancellation schemes are the relative power levels of the various SI terms. However, as already mentioned, the RF cancellation performance is likely to differ between these techniques, and also the hardware and computational requirements are different for each RF canceller structure.

In the forthcoming analysis, we will refer to the parameters of the signal model in all cases by $\breve{h}_{i, p, \mathbf{s}^{k}}(m)$, similar to the above derivations, even though the exact values of the different coefficients vary for different RF cancellation techniques. This notation will simplify the equations and make them more straightforward and illustrative. Hence, the signal after RF cancellation, which is then processed by the digital canceller, can be written as

$$
\begin{aligned}
r_{i}(n)= & \sum_{\substack{p=1 \\
p \text { odd }}}^{P} \sum_{\substack{k \\
\mathbf{s}^{k} \|_{1}=p}} \sum_{m=0}^{M+\max \left(L, L^{\prime}\right)} \breve{h}_{i, p, \mathbf{s}^{k}}(m) \\
& \times \prod_{q=1}^{N_{T}} x_{q}(n-m)^{s_{q}^{k}} x_{q}^{*}(n-m)^{s_{q+N_{T}}^{k},}
\end{aligned}
$$

Note that this signal model implicitly incorporates also the IQ imbalance occurring in the receiver, even though it is omitted in the derivations for brevity [15].

\subsection{Total number of basis functions in the overall model}

In general, with the above cascaded modeling approach for IQ modulator and PA impairments with crosstalk between the transmitters, it can easily be shown that the total number of basis functions in (16) becomes

$$
n_{b}=\sum_{\substack{p=1 \\
p \text { odd }}}^{P}\left(\begin{array}{c}
p+2 N_{T}-1 \\
2 N_{T}-1
\end{array}\right) .
$$

Figure 3 illustrates the number of basis functions for different nonlinearity orders and numbers of transmit antennas for the full signal model and also for the crosstalk-free signal model discussed below in Section 2.4. It is immediately obvious that with higher order MIMO systems, or with heavily nonlinear PAs, the number of basis functions becomes unacceptably high when utilizing the full signal model with crosstalk. Thus, it is necessary to determine methods that will decrease the number of basis functions, and thereby facilitate the estimation of the parameters of this signal model also in practice.

Luckily, many of the terms arising from the cascade of the impairments are so insignificant that they can be neglected with very little effect on the overall modeling accuracy. This will reduce the computational cost of such modeling and the corresponding cancellation procedure. In this work, we propose a specific preprocessing stage which can be used to decrease the dimensionality of the full signal model in (16). This is elaborated in more details in Section 3.2.

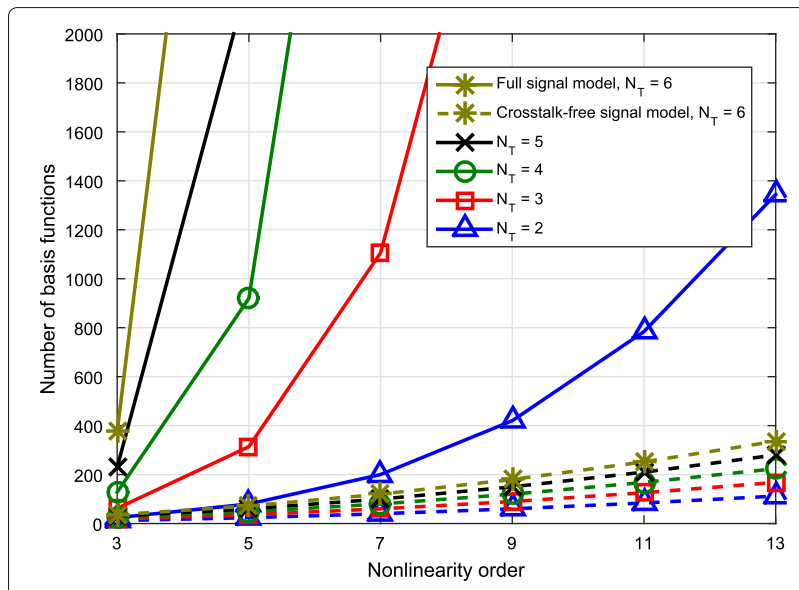

Fig. 3 The number of basis functions with respect to the nonlinearity order of the signal model $(P)$. The curves have been plotted for different numbers of transmit antennas and for both the full signal model and the crosstalk-free signal model 


\subsection{Nonlinear signal model without crosstalk}

Another simple way to decrease the number of basis functions is to neglect the crosstalk effect between the transmitters. Then, the cross terms between the different transmit signals will be removed, which obviously results in a significant decrease in the number of unknown parameters. Modifying (16) accordingly, we can write the signal model now as

$$
\begin{aligned}
r_{i}(n)= & \sum_{j=1}^{N_{T}} \sum_{\substack{p=1 \\
p \text { odd }}}^{P} \sum_{q=0}^{p} \sum_{m=0}^{M+\max \left(L, L^{\prime}\right)} \breve{h}_{i, j, p, q}(m) \\
& \times x_{j}(n-m)^{q} x_{j}^{*}(n-m)^{p-q},
\end{aligned}
$$

where $\breve{h}_{i, j, p, q}(m)$ represents now the coupling channel corresponding to the considered SI signal terms propagating from the $j$ th transmitter to the $i$ th receiver. This signal model is also derived in [15], where it is briefly discussed and analyzed. For this reason, the detailed derivation process of (18) is omitted in this article.

Since now all the cross-terms are neglected from the signal model, the number of basis functions can be expressed as

$$
n_{b}^{C T-\text { free }}=N_{T}\left(\frac{P+1}{2}\right)\left(\frac{P+1}{2}+1\right) .
$$

When investigating Fig. 3, it can be seen that this signal model results in a significant reduction of basis functions, when compared to the full signal model with crosstalk. With moderate crosstalk levels, it is therefore likely that using this signal model will provide a very favorable tradeoff between cancellation performance and computational complexity. However, as already discussed, in highly integrated transceivers explicit modeling of the crosstalk between the transmitters is likely required in order to ensure sufficient cancellation performance [28].

\section{Self-interference parameter estimation and digital cancellation}

In this section, building on the previous modeling in, e.g., $[15,29]$, we will describe the proposed digital cancellation algorithm that models both IQ imbalance and PA nonlinearity in a MIMO full-duplex transceiver with crosstalk between the transmitters. In general, there are two possible approaches for nonlinear digital SI cancellation: (i) construct a linear-in-parameters model of the observed SI signal in the digital domain, including the different impairments, the MIMO propagation channel, and RF cancellation, estimate the unknown parameters of the model, and finally recreate and cancel the SI from the received signals; (ii) have separate models for the MIMO propagation channel and the transmitter impairments, estimate the unknown model parameters sequentially, and recreate and cancel the SI from the received signals. Typically the latter approach is computationally less demanding, but it requires a more elaborate estimation procedure. In this article, we consider the former approach, while the latter is left for future work.

\subsection{Linear-in-parameters model}

Having already derived a linear-in-parameters signal model in Section 2, presented in (16), the next step is to estimate its parameters in $\breve{h}_{i, p, \mathbf{s}^{k}}(m)$. After this, the estimated parameters are used to regenerate the SI signals, which are then subtracted from the received signals at digital baseband to obtain cancellation. Figure 4 shows the whole digital cancellation procedure on a fundamental level.

Denoting the desired signal of interest and additive noise at the $i$ th receiver by $s_{i}(n)$ and $w_{i}(n)$, respectively, the overall received signal at digital baseband can be expressed as

$$
y_{i}(n)=r_{i}(n)+s_{i}(n)+w_{i}(n) .
$$

The corresponding output of the digital SI canceller is then

$$
\hat{s}_{i}(n)=y_{i}(n)-\hat{r}_{i}(n),
$$

where $\hat{r}_{i}(n)$ denotes the SI estimate obtained using the signal model in (16) with estimated parameters, written as

$$
\begin{aligned}
\hat{r}_{i}(n)= & \sum_{\substack{p=1 \\
p \text { odd }}}^{\bar{P}} \sum_{\substack{k \\
\mathbf{s}^{k} \|_{1}=p}} \sum_{m=-M_{1}}^{M_{2}} \hat{\breve{h}}_{i, p, \mathbf{s}^{k}}(m) \\
& \times \prod_{q=1}^{N_{T}} x_{q}(n-m)^{s_{q}^{k}} x_{q}^{*}(n-m)^{s_{q+N_{T}}^{k}} .
\end{aligned}
$$

Here, $\bar{P}$ is the nonlinearity order of the digital canceller, $M_{1}$ is the number of pre-cursor taps, $M_{2}$ is the number of post-cursor taps, and $\hat{\breve{h}}_{i, p, \mathbf{s}^{k}}(m)$ contains the estimated parameters of the signal model. The pre-cursor taps are

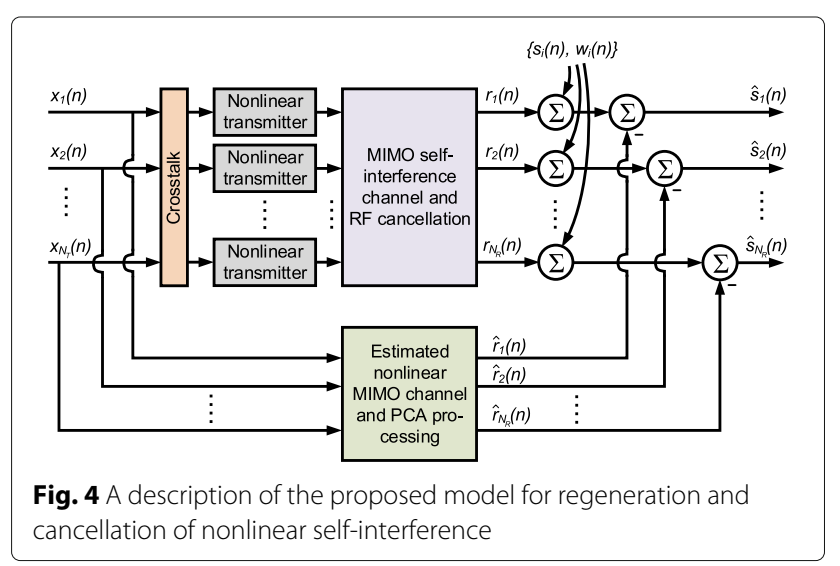


introduced to model all the memory effects produced by the transmitter and RF cancellation circuitry.

\subsubsection{Least-squares-based estimator}

In this work, the actual parameter learning is performed with the widely used least squares (LS) estimation. For brevity, the parameter learning and digital cancellation procedure is here outlined only for the $i$ th receiver, since the procedure is identical for all the receivers.

In practice, calculating the LS estimate requires knowledge of (i) the original transmitted data signal, (ii) the predetermined signal model in (16), and (iii) the observed received signal $y_{i}(n)$. In the considered MIMO full-duplex device, all of these are obviously known by the digital canceller. Since the LS estimation is performed using a block of data, the vector/matrix representations of the relevant signals with $N$ observed samples are first defined as

$$
\begin{aligned}
& \mathbf{y}_{i}=\mathbf{r}_{i}+\mathbf{s}_{i}+\mathbf{w}_{i} \text {, with } \\
& \mathbf{y}_{i}=\left[y_{i}(n) y_{i}(n+1) \cdots y_{i}(n+N-1)\right]^{\mathrm{T}}
\end{aligned}
$$

and $\mathbf{r}_{i}, \mathbf{s}_{i}, \mathbf{w}_{i}$ are defined in the same manner as $\mathbf{y}_{i}$. The error vector is then defined as

$$
\mathbf{e}_{i}=\mathbf{y}_{i}-\hat{\mathbf{r}}_{i}
$$

where the nonlinear SI estimate is

$$
\hat{\mathbf{r}}_{i}=\boldsymbol{\Psi} \hat{\mathbf{h}}_{i}
$$

Here, $\boldsymbol{\Psi}$ is a horizontal concatenation of the convolution matrices defined as follows:

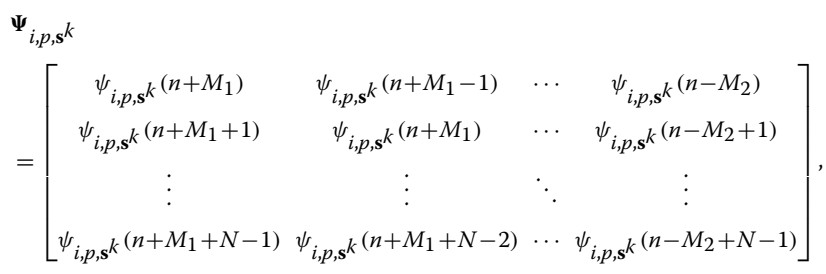

where

$$
\psi_{i, p, \mathbf{s}^{k}}(n)=\prod_{q=1}^{N_{T}} x_{q}(n)^{\mathbf{s}_{q}^{k}} x_{q}^{*}(n)^{\mathbf{s}_{q+N_{T}}^{k},}
$$

with $p=1,3, \ldots, \bar{P}$, and $\mathbf{s}^{k}$ is each combination for which $\left\|\mathbf{s}^{k}\right\|_{1}=p$, similar to the sum limits shown in (16). Overall, the number of concatenated matrices is given by the total number of basis functions in (17), since this is the amount of different combinations of $\mathbf{s}^{k}$ for all the nonlinearity orders.
Alternatively, in the crosstalk-free model $\boldsymbol{\Psi}$ consists of the concatenation of the matrices defined as follows:

$$
\begin{aligned}
& \boldsymbol{\Psi}_{j, p, q} \\
& =\left[\begin{array}{cccc}
\psi_{j, p, q}\left(n+M_{1}\right) & \psi_{j, p, q}\left(n+M_{1}-1\right) & \cdots & \psi_{j, p, q}\left(n-M_{2}\right) \\
\psi_{j, p, q}\left(n+M_{1}+1\right) & \psi_{j, p, q}\left(n+M_{1}\right) & \cdots & \psi_{j, p, q}\left(n-M_{2}+1\right) \\
\vdots & \vdots & \ddots & \vdots \\
\psi_{j ;, p, q}\left(n+M_{1}+N-1\right) & \psi_{j, p, q}\left(n+M_{1}+N-2\right) & \cdots & \psi_{j, p, q}\left(n-M_{2}+N-1\right)
\end{array}\right],
\end{aligned}
$$

where $\psi_{j, p, q}(n)=x_{j}(n)^{q} x_{j}^{*}(n)^{p-q}$, with $j=1,2, \ldots, N_{T}$, $p=1,3, \ldots, \bar{P}$, and $q=0,1, \ldots, p$.

An estimate of the parameter vector $\breve{\mathbf{h}}_{i}$, denoted by $\hat{\breve{h}}_{i}$, is a vertical concatenation of the vectors

$$
\hat{\grave{\mathbf{h}}}_{i, p, \mathbf{s}^{k}}=\left[\hat{\breve{h}}_{i, p, \mathbf{s}^{k}}\left(-M_{1}\right) \cdots \hat{\breve{h}}_{i, p, \mathbf{s}^{k}}\left(M_{2}\right)\right]^{T}
$$

In the crosstalk-free model, the parameter vector consists of the concatenation of vectors

$$
\hat{\breve{h}}_{i, j, p, q}=\left[\hat{\breve{h}}_{i, j, p, q}\left(-M_{1}\right) \cdots \hat{\breve{h}}_{i, j, p, q}\left(M_{2}\right)\right]^{T}
$$

The LS estimate of the parameter vector $\breve{\mathbf{h}}_{i}$ is then found as the solution which minimizes the power of the error vector $\mathbf{e}_{i}$, as

$$
\begin{aligned}
\hat{\breve{\mathbf{h}_{i}}} & =\underset{\breve{\mathbf{h}}_{i}}{\arg \min }\left\|\mathbf{e}_{i}\right\|^{2}=\underset{\breve{\mathbf{h}}_{i}}{\arg \min }\left\|\mathbf{y}_{i}-\boldsymbol{\Psi} \breve{\mathbf{h}}_{i}\right\|^{2} \\
& =\left(\boldsymbol{\Psi}^{H} \boldsymbol{\Psi}\right)^{-1} \boldsymbol{\Psi}^{H} \mathbf{y}_{i},
\end{aligned}
$$

assuming full column rank in $\Psi$.

\subsection{Computationally efficient estimation with principal component analysis}

Another approach to simplify the estimation procedure is to retain the cross-terms, and instead determine which of them are actually significant in terms of the cancellation performance. In this analysis, principal component analysis (PCA) [41] is used to decrease the number of parameters to be estimated. The idea behind the PCA is to determine which of the terms have the highest variance, providing valuable information regarding the significance of the different basis functions. In practice, PCA results in a transformation matrix, with which the original data matrix is multiplied. The size of the transformation matrix can be chosen to provide the desired number of parameters for the final estimation procedure.

There are also various alternative solutions for model complexity reduction, such as compressed sampling (CS) based techniques. Nevertheless, in this work, we choose to use the PCA since it is a straight-forward method for the complexity reduction of the proposed signal model, while also providing nearly the same performance as CS when high modeling accuracy is required [42]. Experimenting with different complexity reduction methods is an important future work item for us. 
The first step in obtaining the desired PCA transformation matrix is to determine the least squares channel estimate given in (28) using all the basis functions. This estimate should be calculated with the highest possible transmit power, since the nonlinear SI terms that are negligible with the highest power will also be negligible with any lower transmit power. Hence, this reveals the terms, which can be omitted under the whole considered transmit power range. If the transceiver in question has more than one receiver chain, the channel estimation can be done individually for all of them, after which the mean value of the estimates is calculated. This is done to avoid having separate transformation matrices for each receiver, resulting in a decreased amount of required data storage. The hereby obtained coefficient vector, which is denoted by $\hat{\mathbf{h}}_{0}$, is used as an initial channel estimate for the full set of basis functions.

The next step is to determine the relative strengths of the different terms present in the SI signal. Using the initial channel estimate, this can be done by multiplying the original data matrix with the obtained estimate. Then, we get

$$
\boldsymbol{\Psi}_{0}=\left(\hat{\mathbf{1}}_{0}^{T}\right) \times \boldsymbol{\Psi}
$$

where $\mathbf{1}$ is a column vector consisting of $1 \mathrm{~s}$, and $\times$ denotes element-wise multiplication between two matrices. The matrix $\boldsymbol{\Psi}_{0}$ now contains all the SI terms in its columns, each multiplied with the corresponding coefficient of the initial channel estimate.

As a starting point for the PCA, the singular value decomposition of the normalized data matrix can be expressed as

$$
\boldsymbol{\Psi}_{0}=\mathbf{U} \Sigma \mathbf{V}^{H},
$$

where $\mathbf{U}$ and $\mathbf{V}$ are the matrices containing the left and right singular vectors, respectively, while $\Sigma$ is a diagonal matrix consisting of the corresponding singular values. In this analysis, it is assumed that the singular values are in decreasing order. To minimize the possible numerical issues upon the PCA transformation, the actual transformation matrix is obtained in its normalized form, which is given by

$$
\mathbf{W}=\mathbf{V} \Sigma^{-1} \text {. }
$$

To control the number of parameters, part of the columns of the obtained matrix $\mathbf{W}$ can then be omitted. Based on the earlier assumption regarding the ordering of the singular values, the columns of the transformation matrix represent the different parameters in the descending order of their significance. Thus, by starting to remove the columns from the right, the number of parameters can be decreased with minimal effect on the modeling accuracy. Thus, denoting the number of chosen parameters with $u$, we can write the final transformation matrix as

$$
\widetilde{\mathbf{W}}=\left[\begin{array}{llll}
\mathbf{w}_{1} & \mathbf{w}_{2} & \cdots & \mathbf{w}_{u}
\end{array}\right],
$$

where $\mathbf{w}_{i}$ is the $i$ th column of the matrix $\mathbf{W}$. Finally, the reduced data matrix can be calculated as

$$
\widetilde{\boldsymbol{\Psi}}=\boldsymbol{\Psi} \widetilde{\mathbf{W}}
$$

The hereby obtained data matrix is then used in the least squares estimation as a replacement for the original data matrix $\Psi$. It should also be noted that when generating the actual digital cancellation signal, the cancellation data matrix must be transformed with the same matrix $\widetilde{\mathbf{W}}$, as the SI channel estimate is only valid in this transformed space.

An important aspect to point out is that the transformation matrix $\widetilde{\mathbf{W}}$ is calculated only once with the highest transmit power, after which it can be used with all transmit powers to reduce the number of basis functions. Namely, since the strengths of the nonlinearities are directly proportional to the transmit power, the SI terms that are negligibly weak with the highest transmit power are at least as weak with the lower transmit powers, which means that the same SI terms can be omitted also then. This is also proven by the waveform simulations, the results of which will be discussed in Section 4. However, should the SI channel change drastically at any point, then the matrix $\widetilde{\mathbf{W}}$ must be recalculated to ensure that no significant memory taps are neglected.

In general, perhaps the most crucial design problem in the context of the PCA is to determine the optimal number of parameters to be included in the final model. This can be most easily determined experimentally by reducing the number of parameters until the obtained cancellation performance starts to drop. Also, the singular values in $\Sigma$ can be used to calculate the percentage of the variance accounted for by the included basis functions. We will address this issue more closely with the help of waveform simulations in Section 4.

\section{Performance simulations and analysis}

The evaluation of the proposed scheme is now done with realistic waveform simulations, utilizing a comprehensive inband full-duplex transceiver model. It incorporates all the relevant impairments, and thereby the SI waveform represents a real-world scenario rather well. Below, we describe the waveform simulator in detail, after which the results are shown. As an important future work item, we aim to evaluate the proposed scheme also with actual RF measurements to confirm the results obtained here with the simulations.

\subsection{Simulation setup and parameters}

The waveform simulations are performed with Matlab, where all the relevant aspects of the full-duplex 
transceiver are modeled. These include the nonlinearity of the PAs, the crosstalk between the transmitters (both before and after the PA), the multipath SI channel, the imperfect RF cancellation, nonlinearity of the receiver, IQ imbalance, phase noise, and the quantization upon analog-to-digital conversion, while the DAC/ADC nonlinearities are omitted also from the simulator model since we have not observed them to be a significant factor in our earlier RF measurements $[3,43]$. This means that the simulator model is rather comprehensive and can be expected to provide realistic results, although they must still be confirmed with real-life measurements. Note that, since the focus of this work is on SI cancellation, the signal of interest is not present in any of the simulations. The RF cancellation is performed in all the cases using the transmitter output signal, since the essential signal model is not affected by the RF cancellation procedure, as shown in Section 2.2. The used waveform is a $20 \mathrm{MHz}$ LTE downlink signal, which utilizes OFDM with a 4QAM constellation. When modeling the phase noise, a common local oscillator for all the transmitters and receivers is assumed, which is a feasible assumption for an inband full-duplex device. All the relevant parameters of the waveform simulator are listed in Table 1, while the used phase noise characteristics are shown in Fig. 5.

In the forthcoming results, five different digital cancellers are considered, and they are as follows:

Table 1 The relevant parameters of the waveform simulator

\begin{tabular}{ll}
\hline Parameter & Value \\
\hline Bandwidth & $20 \mathrm{MHz}$ \\
Sampling frequency & $122.88 \mathrm{MHz}$ \\
Number of TX/RX antennas & $2 / 2$ \\
PA gain & $27 \mathrm{~dB}$ \\
PA IIP3 & $13 \mathrm{dBm}$ \\
Level of TX crosstalk before the PAs & $-10 \mathrm{~dB} /$ varied \\
Level of TX crosstalk after the PAs & $-10 \mathrm{~dB}$ \\
Receiver noise floor & $-96.9 \mathrm{dBm}$ \\
Phase noise characteristics & See Fig. 5 \\
Transmit power & $25 \mathrm{dBm} /$ varied \\
SI channel length & $20 \mathrm{taps}$ \\
Antenna attenuation & $40 \mathrm{~dB}$ \\
RF cancellation & $30 \mathrm{~dB}$ \\
IRR (TX/RX) & $25 \mathrm{~dB}$ \\
ADC bits & 12 \\
Parameter estimation sample size $(N)$ & $30,000 /$ varied \\
Parameter estimation sample size for PCA & 10,000 \\
Nonlinearity order of the canceller $(P)$ & 5 \\
Number of pre-cursor taps $\left(M_{1}\right)$ & 10 \\
Number of post-cursor taps $\left(M_{2}\right)$ & 20 \\
\hline
\end{tabular}

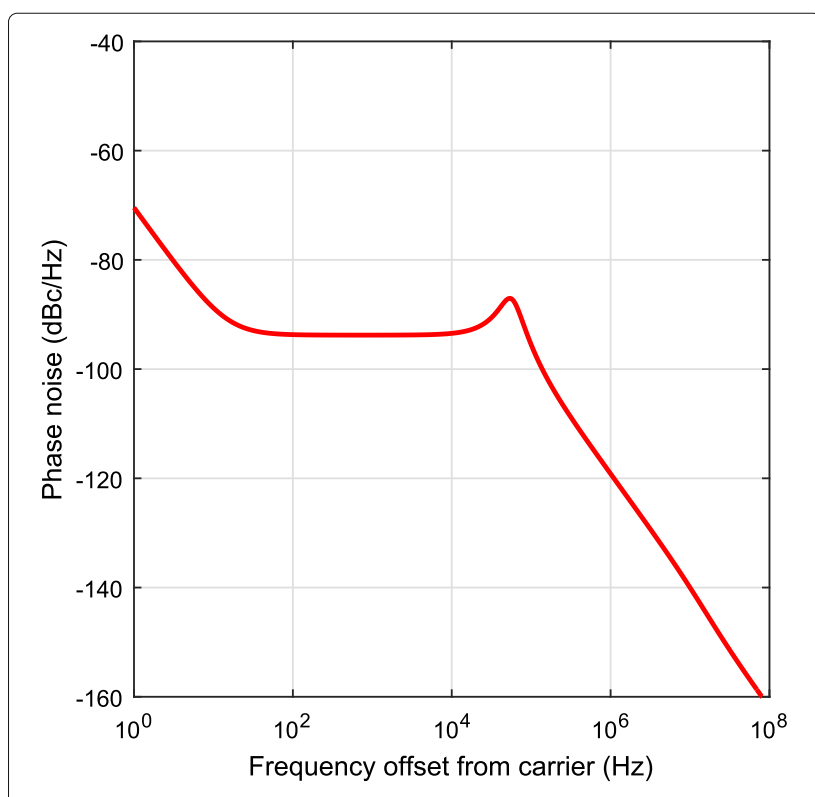

Fig. 5 The phase noise characteristics used in the waveform simulator, corresponding to a charge-pump type phase locked loop-based oscillator, taken from: http://datasheets.maximintegrated.com/en/ds/ MAX2870.pdf

- Digital canceller with the full signal model in (22), including PCA processing to decrease the dimensionality and computational complexity

- Digital canceller with the full signal model in (22), but without any dimensionality reduction

- Digital canceller utilizing the $\mathrm{N}$-input memory model from [26], which considers the nonlinearity of the PA and both linear and nonlinear crosstalk.

- Digital canceller with the crosstalk-free signal model in (18), from [15], where both the nonlinearity of the PA and the IQ imbalance are modeled.

- Digital canceller with a traditional linear signal model, where $P=1$.

In all the cases, the same parameter estimation sample size is used for the different cancellers with $M_{1}=10$ and $M_{2}=20$ to ensure a fair comparison. The PCA matrix is calculated using 10000 samples in the initial channel estimation stage. Furthermore, to avoid overfitting when estimating and cancelling the SI, separate portions of the signal are used for calculating the SI channel estimate and evaluating the actual SI cancellation performance.

\subsection{Results}

First, the signal spectra after the different digital cancellers are shown in Fig. 6 using the default parameters, alongside with the spectra of the RF cancelled signal and the receiver noise floor. It can be observed that only the digital cancellers utilizing the full signal model can obtain 


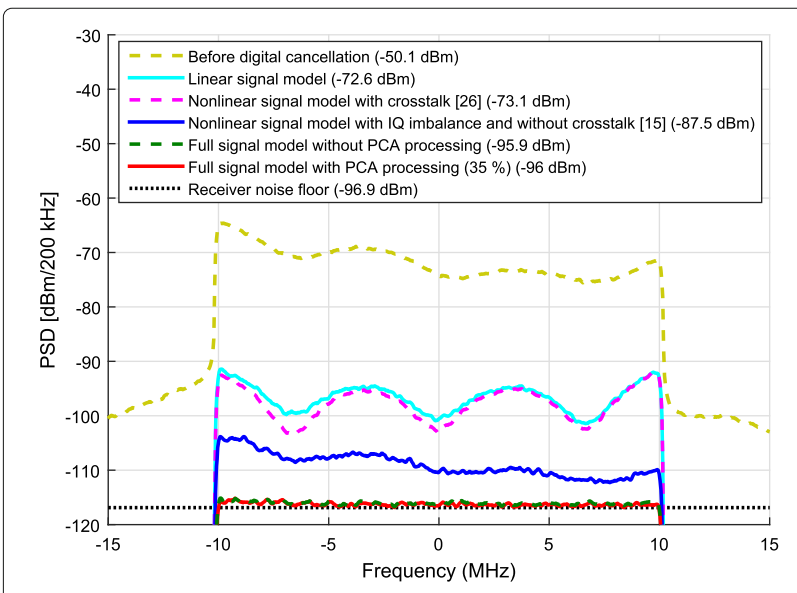

Fig. 6 The signal spectra after the different digital cancellers, alongside with the spectra of the RF cancelled signal and the receiver noise floor

sufficient levels of SI cancellation. In particular, the digital canceller utilizing the linear signal model and the nonlinear crosstalk signal model from [26] perform very poorly since in this case IQ imbalance is the dominant source of distortion. The signal model from [15], on the other hand, has insufficient modeling accuracy since it does not take into account the crosstalk. Thereby, it is necessary to model both the IQ imbalance and the crosstalk, together with the nonlinearity of the PA, to obtain sufficient levels of digital cancellation. Furthermore, based on Fig. 6, the number of basis functions can be reduced to $35 \%$ without any reduction in the cancellation performance when using the full signal model.

Note that in this case the phase noise has no significant effect on the residual SI power since a common local oscillator between the transmitters and receivers is assumed. This results in a certain level of self-cancellation of the phase noise upon downconversion, considerably reducing its significance [44].

Figure 7 shows then the increase in the effective noise floor due to the residual SI for the different digital cancellers, with respect to the total transmit power. In other words, the closer to $0 \mathrm{~dB}$ the canceller achieves, the better is its overall SI cancellation performance. As expected, the linear canceller is not capable of efficient cancellation even with the lowest transmit powers, whereas the nonlinear cancellers with IQ imbalance modeling suppress the SI nearly perfectly up to transmit powers of $20 \mathrm{dBm}$. Moreover, the digital canceller utilizing the nonlinear crosstalk signal model from [26] performs very poorly with the whole transmit power range since it does not model the IQ imbalance, as already discussed.

With transmit powers beyond $20 \mathrm{dBm}$, the crosstalk effects begin to decrease also the accuracy of the crosstalk-free nonlinear signal model from [15]. On the other hand, the full signal models perform relatively well

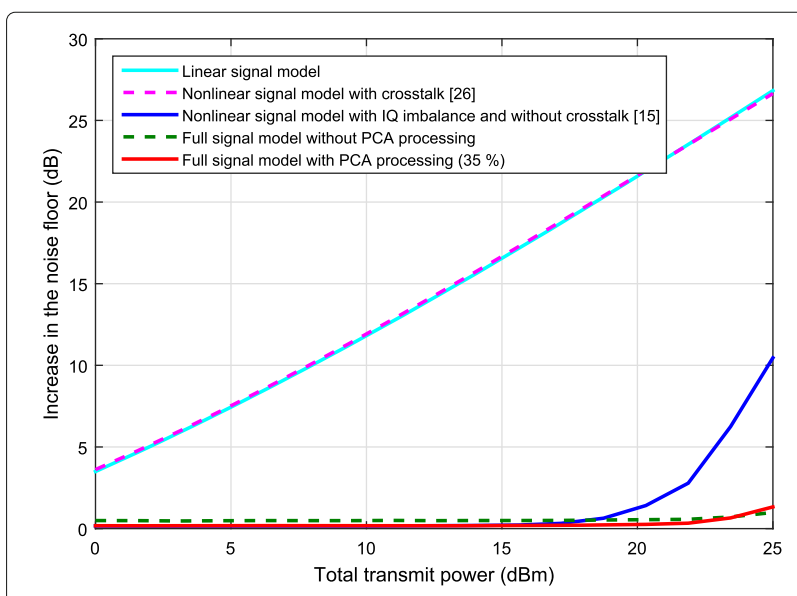

Fig. 7 The increase in the noise floor due to residual SI, with respect to the total transmit power

even with the highest transmit powers, resulting in only a very minor increase in the noise floor. Furthermore, as observed earlier, retaining only $35 \%$ of the terms after the PCA processing does not seem to decrease the accuracy of the signal model when compared to the full signal model with all the terms included. In fact, the performance of the digital canceller with the lower transmit powers is slightly improved by the dimensionality reduction since the smaller number of parameters results in a more accurate parameter vector estimate, and hence in more efficient cancellation.

To investigate the PCA-based dimensionality reduction in greater detail, Fig. 8 shows the increase in the noise floor with respect to the percentage of the terms included after the PCA, when using the full signal model in (22). The performance of the case without any PCA processing is also shown for reference. It can be observed from the

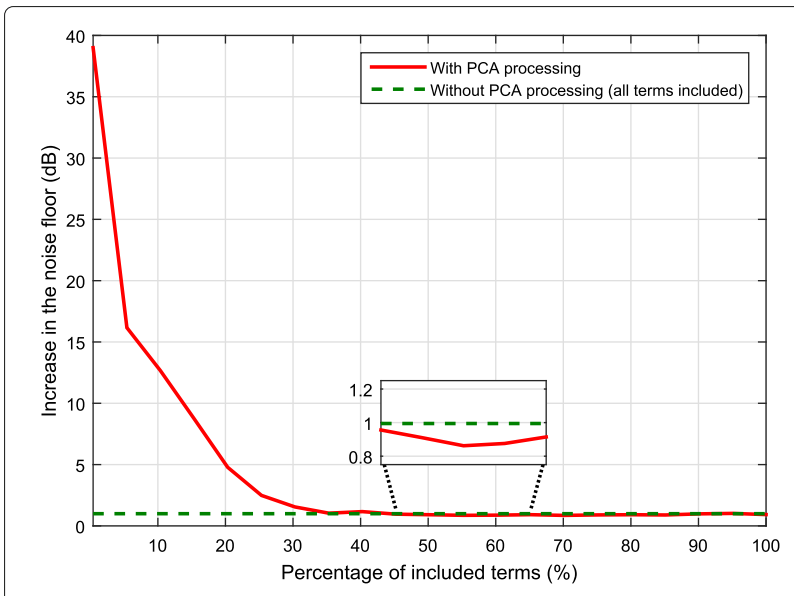

Fig. 8 The increase in the noise floor due to residual SI, with respect to the percentage of included terms 
figure that there is a wide range of values for the percentage of included terms that provide the same cancellation performance. However, if the percentage of included terms goes significantly below $35 \%$, the performance of the PCA-based canceller is rather poor. This is caused by the decreased accuracy of the signal model due to excluding some of the significant terms. Also note that when $50-80 \%$ of the terms are included, the PCA-based solution achieves slightly higher levels of SI cancellation than the canceller without PCA processing. The reason for this is the decreased variance of the parameter estimate, thanks to the smaller number of terms.

In order to minimize the computational complexity of the cancellation procedure, the number of included terms must obviously be minimized. Hence, the smallest number of terms that still provides the required performance is in this sense the optimal choice. Figure 8 indicates that, with the parameters considered in these simulations, the optimal percentage of included terms is roughly $35 \%$, which corresponds to 840 coefficients with the considered nonlinearity order and number of memory taps.

Since the level of the crosstalk occurring before the transmitter PAs is obviously the most significant aspect in determining whether the full signal model is actually necessary, Fig. 9 shows then the performance of the different digital cancellers with different crosstalk levels. It can be observed that, with the considered transmit power of $25 \mathrm{dBm}$, the crosstalk has a rather significant effect already at the level of $-20 \mathrm{~dB}$, since using the nonlinear signal model without any crosstalk modeling from [15] results in a $3 \mathrm{~dB}$ higher noise floor than when using the full signal models. With higher crosstalk levels, the performance difference is obviously further emphasized. Furthermore, similar to the earlier observations, the signal models that do not model the IQ imbalance perform very poorly since it is the dominant source of distortion.

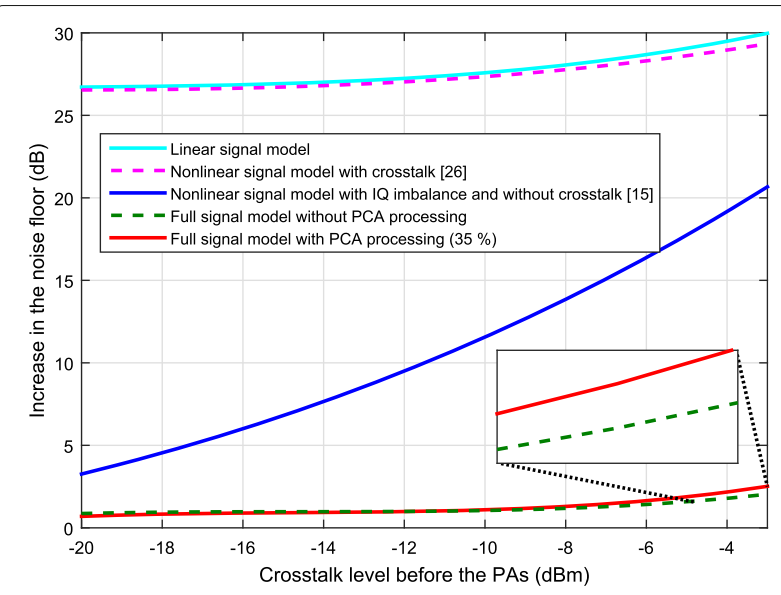

Fig. 9 The increase in the noise floor due to residual SI, with respect to the level of the crosstalk before the PAs
It can also be observed from Fig. 9 that a larger number of terms is required with the very high crosstalk levels. In particular, having only $35 \%$ of the terms retained results in a somewhat higher residual SI power than retaining all of the terms. This is explained by the fact that higher crosstalk levels also result in a larger number of significantly powerful SI terms. Nevertheless, the cancellation performance differences between the full signal models, with or without PCA processing, are still relatively small with these reasonable crosstalk levels.

In order to further investigate the differences in the computational complexity of the different digital cancellers, Fig. 10 shows their performance for different parameter estimation sample sizes $(N)$. It can be observed that the signal models without sufficient modeling accuracy are not bottlenecked by the amount of available learning data, since their performance is largely unaffected by the value of $N$. The benefits of the PCA-based dimensionality reduction for the full signal model are also clearly apparent, since the case with $35 \%$ of the terms retained performs relatively well even with very small parameter estimation sample sizes. As opposed to this, without any dimensionality reduction, roughly $N=$ 24000 is required to obtain a sufficiently accurate estimate of the parameters. Overall, it is hence clear that the PCA processing helps in significantly reducing the computational complexity of the digital SI cancellation procedure when utilizing the full signal model.

\section{Conclusions}

In this paper, a novel digital self-interference canceller for a nonlinear MIMO inband full-duplex transceiver was presented. The canceller is based on a comprehensive signal model for the SI observed in the digital domain, which includes the effect of crosstalk occurring between the transmit chains, while also incorporating the most

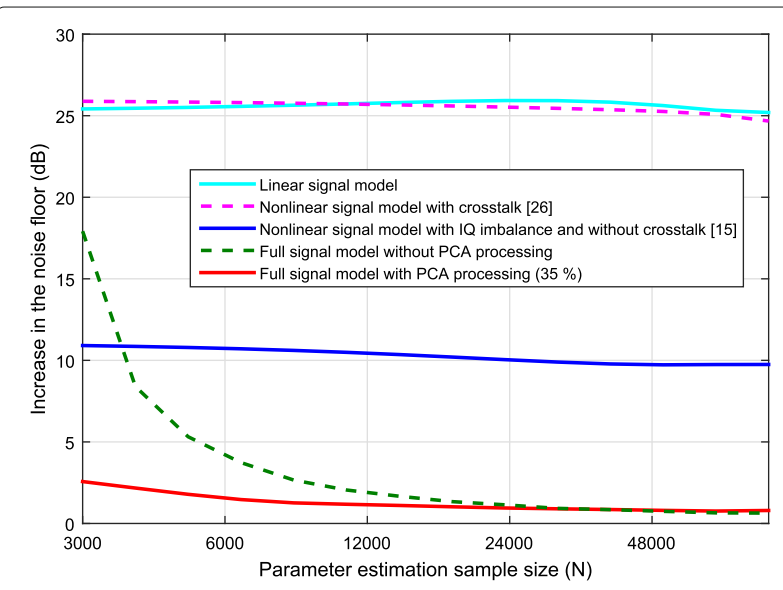

Fig. 10 The increase in the noise floor due to residual $\mathrm{SI}$, with respect to the parameter estimation sample size $(N)$ 
significant RF imperfections. Furthermore, it was also shown that the signal model is valid for various different RF cancellers. To control the complexity of the cancellation procedure, a novel principal component analysis based scheme was then proposed, which can be used to control the number of parameters in the signal model. With the help of waveform simulations, the proposed digital canceller was shown to cancel the SI nearly perfectly, even when its computational complexity was significantly reduced using principal component analysis.

\section{Appendix: Power amplifier output signal under crosstalk}

Let us define a signal $y(n)$ as follows:

$$
y(n)=\sum_{i=1}^{N} \alpha_{i} x_{i}(n),
$$

where $\alpha_{i}$ is a scaling constant and $x_{i}(n)$ are known signals. To express an arbitrary integer power $p$ of the signal $y(n)$ in terms of the signals $x_{i}(n)$, let us expand the corresponding equation accordingly.

$$
y(n)^{p}=\left(\alpha_{1} x_{1}(n)+\sum_{i=2}^{N} \alpha_{i} x_{i}(n)\right)^{p} .
$$

Applying now the binomial theorem to the above expression, we obtain

$$
y(n)^{p}=\sum_{k_{1}=0}^{p}\left(\begin{array}{l}
p \\
k_{1}
\end{array}\right)\left(\alpha_{1} x_{1}(n)\right)^{k_{1}}\left(\sum_{i=2}^{N} \alpha_{i} x_{i}(n)\right)^{p-k_{1}}
$$

Applying the binomial theorem in a similar manner to the expression $\left(\sum_{i=2}^{N} \alpha_{i} x_{i}(n)\right)^{p-k_{1}}$, we get

$$
\begin{aligned}
y(n)^{p}= & \sum_{k_{1}=0}^{p}\left[\left(\begin{array}{l}
p \\
k_{1}
\end{array}\right)\left(\alpha_{1} x_{1}(n)\right)^{k_{1}}\right. \\
& \left.\times\left(\alpha_{2} x_{2}(n)+\sum_{i=3}^{N} \alpha_{i} x_{i}(n)\right)^{p-k_{1}}\right] \\
= & \sum_{k_{1}=0}^{p}\left[( \begin{array} { c } 
{ p } \\
{ k _ { 1 } }
\end{array} ) ( \alpha _ { 1 } x _ { 1 } ( n ) ) ^ { k _ { 1 } } \left[\sum _ { k _ { 2 } = 0 } ^ { p - k _ { 1 } } \left[\left(\begin{array}{c}
p-k_{1} \\
k_{2}
\end{array}\right)\right.\right.\right. \\
& \left.\left.\left.\times\left(\alpha_{2} x_{2}(n)\right)^{k_{2}}\left(\sum_{i=3}^{N} \alpha_{i} x_{i}(n)\right)^{p-k_{1}-k_{2}}\right]\right]\right] \\
= & \sum_{k_{1}=0}^{p} \sum_{k_{2}=0}^{p-k_{1}}\left(\begin{array}{c}
p \\
k_{1}
\end{array}\right)\left(\begin{array}{c}
p-k_{1} \\
k_{2}
\end{array}\right)\left(\alpha_{1} x_{1}(n)\right)^{k_{1}} \\
& \times\left(\alpha_{2} x_{2}(n)\right)^{k_{2}}\left(\sum_{i=3}^{N} \alpha_{i} x_{i}(n)\right)^{p-k_{1}-k_{2}} .
\end{aligned}
$$

Applying the binomial theorem again to the expression $\left(\sum_{i=3}^{N} \alpha_{i} x_{i}(n)\right)^{p-k_{1}-k_{2}}$ and continuing in a similar manner, we finally obtain the following equation:

$$
\begin{aligned}
\left(\sum_{i=1}^{N} \alpha_{i} x_{i}(n)\right)^{p}= & \sum_{k_{1}=0}^{p} \sum_{k_{2}=0}^{p-k_{1}} \ldots \sum_{k_{N-1}=0}^{p-k_{1}-\cdots-k_{N-2}}\left(\begin{array}{c}
p \\
k_{1}
\end{array}\right) \\
& \times\left(\begin{array}{c}
p-k_{1} \\
k_{2}
\end{array}\right) \cdots\left(\begin{array}{c}
p-k_{1}-\cdots-k_{N-2} \\
k_{N-1}
\end{array}\right) \\
& \times x_{1}(n)^{k_{1}} x_{2}(n)^{k_{2}} \cdots x_{N}(n)^{p-k_{1}-\cdots-k_{N-1}} \\
= & \sum_{k_{1}=0}^{p} \sum_{k_{2}=0}^{p-k_{1}} \ldots \sum_{k_{N-1}=0}^{p-k_{1}-\cdots-k_{N-2}} A_{k_{1}, \ldots, k_{N-1}} \\
& \times x_{1}(n)^{k_{1}} x_{2}(n)^{k_{2}} \cdots x_{N}(n)^{p-k_{1}-\cdots-k_{N-1},}
\end{aligned}
$$

where $A_{k_{1}, \ldots, k_{N-1}}$ is a constant.

\section{Acknowledgements}

The research work leading to these results was funded by the Academy of Finland (under the projects \#259915, \#301820, and \#304147), the Finnish Funding Agency for Technology and Innovation (Tekes, under the TAKE-5 project), Tampere University of Technology Graduate School, Nokia Foundation, Tuula and Yrjö Neuvo Research Fund, and Emil Aaltonen Foundation.

\section{Competing interests}

The authors declare that they have no competing interests.

Received: 30 June 2016 Accepted: 3 January 2017

Published online: 02 February 2017

\section{References}

1. M Duarte, C Dick, A Sabharwal, Experiment-driven characterization of full-duplex wireless systems. IEEE Trans. Wirel. Commun. 11(12), 4296-4307 (2012)

2. M Heino, D Korpi, T Huusari, E Antonio-Rodríguez, S Venkatasubramanian, T Riihonen, L Anttila, C Icheln, K Haneda, R Wichman, M Valkama, Recent advances in antenna design and interference cancellation algorithms for in-band full-duplex relays. IEEE Commun. Mag. 53(5), 91-101 (2015)

3. D Korpi, Y-S Choi, T Huusari, S Anttila, L Talwar, M Valkama, in Proc. IEEE Global Communications Conference (GLOBECOM). Adaptive nonlinear digital self-interference cancellation for mobile inband full-duplex radio: algorithms and RF measurements (Institute of Electrical and Electronics Engineers (IEEE), New York, 2015)

4. T Riihonen, S Werner, R Wichman, Mitigation of loopback self-interference in full-duplex MIMO relays. IEEE Trans. Signal Process. 59(12), 5983-5993 (2011)

5. Jl Choi, M Jain, K Srinivasan, P Levis, S Katti, in Proc. 16th Annual International Conference on Mobile Computing and Networking. Achieving single channel full duplex wireless communication (Association for Computing Machinery (ACM), New York, 2010), pp. 1-12

6. M Jain, JI Choi, T Kim, D Bharadia, S Seth, K Srinivasan, P Levis, S Katti, P Sinha, in Proc. 17th Annual International Conference on Mobile Computing and Networking. Practical, real-time, full duplex wireless (Association for Computing Machinery (ACM), New York, 2011), pp. 301-312

7. D Bharadia, S Katti, in Proc. 11th USENIX Conference on Networked Systems Design and Implementation. Full duplex MIMO radios (USENIX, the Advanced Computing Systems Association, Berkeley, 2014), pp. 359-372

8. A Sabharwal, P Schniter, D Guo, D Bliss, S Rangarajan, R Wichman, In-band full-duplex wireless: Challenges and opportunities. IEEE J. Sel. Areas Commun. 32(9), 1637-1652 (2014) 
9. Y-S Choi, H Shirani-Mehr, Simultaneous transmission and reception: algorithm, design and system level performance. IEEE Trans. Wirel. Commun. 12(12), 5992-6010 (2013)

10. E Everett, A Sahai, A Sabharwal, Passive self-interference suppression for full-duplex infrastructure nodes. IEEE Trans. Wirel. Commun. 13(2), 680-694 (2014)

11. G Liu, FR Yu, H Ji, VCM Leung, X Li, In-band full-duplex relaying: a survey, research issues and challenges. IEEE Commun. Surv. Tutorials. 17(2), 500-524 (2015)

12. Z Zhang, Y Shen, S Shao, W Pan, Y Tang, in Proc. Sixth International Conference on Wireless Communications and Signal Processing (WCSP). Full duplex 2×2 MIMO radios (Institute of Electrical and Electronics Engineers (IEEE), New York, 2014), pp. 1-6

13. A Balatsoukas-Stimming, P Belanovic, K Alexandris, A Burg, in Proc. 47th Asilomar Conference on Signals, Systems and Computers. On self-interference suppression methods for low-complexity full-duplex MIMO (Institute of Electrical and Electronics Engineers (IEEE), New York, 2013), pp. 992-997

14. K Alexandris, A Balatsoukas-Stimming, A Burg, in Proc. IEEE 8th Sensor Array and Multichannel Signal Processings Workshop (SAM). Measurement-based characterization of residual self-interference on a full-duplex MIMO testbed (Institute of Electrical and Electronics Engineers (IEEE), New York, 2014), pp. 329-332

15. L Anttila, D Korpi, E Antonio-Rodríguez, R Wichman, M Valkama, in Proc. IEEE Globecom Workshops. Modeling and efficient cancellation of nonlinear self-interference in MIMO full-duplex transceivers (Institute of Electrical and Electronics Engineers (IEEE), New York, 2014), pp. 862-868

16. BP Day, AR Margetts, DW Bliss, P Schniter, Full-duplex bidirectional MIMO: achievable rates under limited dynamic range. IEEE Trans. Signal Process. 60(7), 3702-3713 (2012)

17. Z Zhang, Z Chen, M Shen, B Xia, Spectral and energy efficiency of multipair two-way full-duplex relay systems with massive MIMO. IEEE J. Sel. Areas Commun. 34(4), 848-863 (2016)

18. XXia, Y Xu, K Xu, D Zhang, W Ma, Full-duplex massive MIMO AF relaying with semiblind gain control. IEEE Trans. Veh. Technol. 65(7), 5797-5804 (2016)

19. XXia, D Zhang, KXu, W Ma, Y Xu, Hardware impairments aware transceiver for full-duplex massive MIMO relaying. IEEE Trans. Signal Process. 63(24), 6565-6580 (2015)

20. T Huusari, Y-S Choi, P Liikkanen, D Korpi, S Talwar, M Valkama, in Proc. IEEE 81st Vehicular Technology Conference (VTC Spring). Wideband self-adaptive RF cancellation circuit for full-duplex radio: operating principle and measurements (Institute of Electrical and Electronics Engineers (IEEE), New York, 2015)

21. HQ Ngo, HA Suraweera, M Matthaiou, EG Larsson, Multipair full-duplex relaying with massive arrays and linear processing. IEEE J. Sel. Areas Commun. 32(9), 1721-1737 (2014)

22. R Zayani, R Bouallegue, D Roviras, in Proc. 21st Annual IEEE International Symposium on Personal, Indoor and Mobile Radio Communications. Crossover neural network predistorter for the compensation of crosstalk and nonlinearity in MIMO OFDM systems (Institute of Electrical and Electronics Engineers (IEEE), New York, 2010), pp. 966-970

23. S Amin, PN Landin, P Händel, D Rönnow, Behavioral modeling and linearization of crosstalk and memory effects in RF MIMO transmitters. IEEE Trans. Microw. Theory Tech. 62(4), 810-823 (2014)

24. T Sadeghpour, RA Alhameed, NT Ali, ITE Elfergani, Y Dama, OO Anoh, in Proc. 18th IEEE International Conference on Electronics, Circuits and Systems (ICECS). Linear and nonlinear crosstalk in MIMO OFDM transceivers (Institute of Electrical and Electronics Engineers (IEEE), New York, 2011), pp. 504-507

25. J Qi, S Aïssa, in Proc. IEEE Wireless Communications and Networking Conference. Analysis and compensation for the joint effects of HPA nonlinearity, I/Q imbalance and crosstalk in MIMO beamforming systems (Institute of Electrical and Electronics Engineers (IEEE), New York, 2011), pp. 1562-1567

26. D Saffar, N Boulejfen, FM Ghannouchi, A Gharsallah, M Helaoui, Behavioral modeling of MIMO nonlinear systems with multivariable polynomials. IEEE Trans. Microw. Theory Tech. 59(11), 2994-3003 (2011)

27. MV Amiri, SA Bassam, M Helaoui, FM Ghannouchi, in Proc. 15th IEEE International Workshop on Computer Aided Modeling, Analysis and Design of Communication Links and Networks (CAMAD). Matrix-based orthogonal polynomials for MIMO transmitter linearization (Institute of Electrical and Electronics Engineers (IEEE), New York, 2010), pp. 57-60

28. A Abdelhafiz, L Behjat, FM Ghannouchi, M Helaoui, O Hammi, A high-performance complexity reduced behavioral model and digital predistorter for MIMO systems with crosstalk. IEEE Trans. Commun. 64(5), 1996-2004 (2016)

29. L Anttila, D Korpi, Syrjälä, M Valkama, in Proc. 47th Asilomar Conference on Signals, Systems and Computers. Cancellation of power amplifier induced nonlinear self-interference in full-duplex transceivers (Institute of Electrical and Electronics Engineers (IEEE), New York, 2013), pp. 1193-1198

30. D Korpi, L Anttila, Syrjälä, M Valkama, Widely linear digital self-interference cancellation in direct-conversion full-duplex transceiver. IEEE J. Sel. Areas Commun. 32(9), 1674-1687 (2014)

31. A Balatsoukas-Stimming, A Austin, P Belanovic, A Burg, Baseband and RF hardware impairments in full-duplex wireless systems: experimental characterisation and suppression. EURASIP J. Wireless Commun. Netw. 2015(142), 142 (2015)

32. D Korpi, T Huusari, Y-S Choi, L Anttila, S Talwar, M Valkama, in Proc. IEEE 16th International Workshop on Signal Processing Advances in Wireless Communications (SPAWC). Digital self-interference cancellation under nonideal RF components: advanced algorithms and measured performance (Institute of Electrical and Electronics Engineers (IEEE), New York, 2015), pp. 286-290

33. L Anttila, Digital front-end signal processing with widely-linear signal models in radio devices, PhD thesis, Tampere University of Technology. (Tampere University of Technology, Tampere, 2011). http://urn.fi/URN:ISBN:978952-15-2978-8

34. M Isaksson, D Wisell, D Ronnow, A comparative analysis of behavioral models for RF power amplifiers. IEEE Trans. Microw. Theory Technol. 54(1), 348-359 (2006)

35. L Ding, GT Zhou, DR Morgan, Z Ma, JS Kenney, J Kim, CR Giardina, A robust digital baseband predistorter constructed using memory polynomials. IEEE Trans. Commun. 52(1), 159-165 (2004)

36. L Anttila, P Handel, M Valkama, Joint mitigation of power amplifier and I/Q modulator impairments in broadband direct-conversion transmitters. IEEE Trans. Microw. Theory Technol. 58(4), 730-739 (2010)

37. H Ku, JS Kenney, Behavioral modeling of nonlinear RF power amplifiers considering memory effects. IEEE Trans. Microw. Theory Technol. 51(12), 2495-2504 (2003)

38. M Duarte, A Sabharwal, in Proc. 44th Asilomar Conference on Signals, Systems, and Computers. Full-duplex wireless communications using off-the-shelf radios: feasibility and first results (Institute of Electrical and Electronics Engineers (IEEE), New York, 2010), pp. 1558-1562

39. M Duarte, A Sabharwal, V Aggarwal, R Jana, KK Ramakrishnan, CW Rice, NK Shankaranarayanan, Design and characterization of a full-duplex multiantenna system for WiFi networks. IEEE Trans. Veh. Technol. 63(3), 1160-1177 (2014)

40. D Bharadia, E McMilin, S Katti, in Proc. SIGCOMM'13. Full duplex radios (Association for Computing Machinery (ACM), New York, 2013), pp. 375-386

41. IT Jolliffe, Principal component analysis, 2nd Ed. (Springer, New York, 2002)

42. A Abdelhafiz, A Kwan, O Hammi, FM Ghannouchi, Digital predistortion of LTE-A power amplifiers using compressed-sampling-based unstructured pruning of volterra series. IEEE Trans. Microw. Theory Technol. 62(11), 2583-2593 (2014)

43. D Korpi, J Tamminen, M Turunen, T Huusari, Y-S Choi, L Anttila, S Talwar, M Valkama, Full-duplex mobile device: pushing the limits. IEEE Commun. Mag. 54(9), 80-87 (2016)

44. Syrjälä, K Yamamoto, M Valkama, Analysis and design specifications for full-duplex radio transceivers under RF oscillator phase-noise with arbitrary spectral shape. IEEE Trans. Veh. Technol. 65(8), 6782-6788 (2016) 